\title{
Management and planning of water pipeline networks
}

\author{
S. J. Habib ${ }^{1}$, P. N. Marimuthu ${ }^{1}$, S. Kim ${ }^{2}$, Y. $\operatorname{Pan}^{2}$ \& S. X. Yang ${ }^{2}$ \\ ${ }^{1}$ Computer Engineering Department, Kuwait University, Kuwait \\ ${ }^{2}$ Mechanical Engineering Department, \\ Massachusetts Institute of Technology, USA
}

\begin{abstract}
Water is a scare resource in the region of Middle East especially in Kuwait, where it has limited rainfall and natural ground water. Thus, Kuwait depends more than $90 \%$ on non-conventional water sources such as the seawater desalination, whereby the seawater is converted into fresh water through the desalination process. The desalination plants have been proven to supply sufficient amounts of fresh water throughout the years; however, some recent studies in Kuwait show a large amount of water leakages in the water pipeline network (WPN) and intermediate storage units. The wasted amount of water is very high if we consider the cost of producing water through the desalination process that it is roughly around 0.75 to 1.00 dollar per a cubic meter. Also, the up-to-date layout documentation of WPN is unavailable, where this situation poses a challenging problem in monitoring and inspecting WPN. This paper offers three contributions. First, we present a statistical analysis of the water production and consumption and the current status of WPN in Kuwait. Second, we describe an analytical approach to explore WPN based on the analogy between the computer network discovery and WPN discovery, wherein both the networks are viewed as a graph comprising of links and connectors. Third, we propose an integrated WPN discovery system that it deploys smart devices such as mobile robots to discover and validate the layouts of WPN. The manual detection of leakage is limited to surface pipelines, whereas the traditional techniques for passive detection of underground leakage are labor-intensive and very expensive. Thus, the proposed integrated WPN discovery system offers economical, long-term management and planning benefits over the manual inspection.
\end{abstract}

Keywords: capacity planning, network discovery, robot. 


\section{Introduction}

Water resource management is the activity of planning, developing, distributing and managing the optimum use of water resources [1]. Managing water pipeline network (WPN) is a challenging task due to its frequent changes in its topology, which is resulted from new connections and breakages. WPN in Kuwait is a large distribution network transporting adequate supply of water to consumers and industries; moreover, the recent statistical report from Ministry of Electricity and Water (MEW) in the State of Kuwait on existing water pipeline network confirms an average of 2000 new fresh water connections annually. The report explicitly specifies that the water pipe breakages per year reach an average sum of 650 in recent years [2]. This situation envisages the challenges in maintaining WPN and demands the mapping and discovery of WPN, which eases the identification and updating of newly added entities, connection failures and other anomalies in WPN topology.

In general, a structured network is defined as a network comprising of a number of standardized components and subsystems [1]. Computer network is an example of structured network comprising of various entities such as routers, switches, computing units and subnets, whereby, the network topology is frequently altered due to the emerging technological developments and growth of computing nodes. In analogous with the computer network, WPN comprises of reservoirs, pumps, pipelines and control elements and it is also categorized as a structured network. The rapidly growing populations and the development of urban areas cause continuous expansion of WPN, thereby leading to frequent changes in WPN topology. Therefore, the mapping of WPN in its real domain into computer domain eases the periodical updating of WPN's topology, whereby the structure of WPN may be discovered by deriving procedures in analogy with the computer network discovery. Computer network discovery comprehends the existing physical layout by flooding data packets from known entities in the network to the remaining entities, and the discovery lists the interconnections among the network entities. Modern network mapping tools use a lower-level approach, known as the hop-by-hop feedback method for listing all elements within the network, such as nodes, switches, hubs, routers and subnets [3].

In this paper, we report the early stages of developments of an interinstitution research project on developing an integrated WPN discovery system comprising of crawling robots. The contributions of this paper are threefold. First, we have presented a statistical analysis of water consumption and the breakages of WPN in Kuwait for recent years. Second, we have exploited the structural analogy between computer network and water pipeline network to derive an analytical model to explore the WPN topology, wherein both the networks are viewed as a graph comprising of links and connectors. Third, we have discussed the design and the prototype of bio-inspired crawling robot, which is part of the proposed integrated WPN discovery system. 


\section{Existing water pipeline network in Kuwait}

In Kuwait, the water pipeline network comprises of main pumping stations, reservoirs, elevated storage towers, distribution lines and subsidiary networks. The main water pipeline is of pre-stressed concrete cylinder pipes (PCCP 16 bars of $2000 \mathrm{~mm}$ and $1500 \mathrm{~mm}$ diameter). The subsidiary distribution pipes are of Ductile Iron 16 bars (with variable diameters such as $150 \mathrm{~mm}, 300 \mathrm{~mm}, 400 \mathrm{~mm}$, $600 \mathrm{~mm}$ and $800 \mathrm{~mm}$ ), which are internally cement lined and externally coated with Bituminous paint. There are about many valve chambers with butterfly valves, gate valves, air valves and washout valve chambers present in the WPN.

The statistical report from MEW documents the activities of WPN in Kuwait, which shows that the number of consumers and the annual fresh water consumptions are increasing every year [2]. The statistics on number of fresh water consumers for a span of 8 recent consecutive years shows that the number of users is increased by $36.47 \%$. In addition; the number of breakages in WPN starting from year 2007 to year 2011 is presented as a bar chart in Figure 1. It is observed that there was a reduction in the number of breakages in 2008. However, the breakages are found to be increasing from 2009 and it averages out around 265 breakages from 2010. Furthermore, the outcome of a study and analysis conducted by Ghunaimi [4], in 2005 presented the projected growth of water consumption, as illustrated in Figure 2, wherein the average water consumption nearly doubles for every 50 years and it will increase to be more than 6 times within a period of 100 years. The study projects the future growth of water consumption based on the dependency of water consumption with the increase in population in Kuwait.

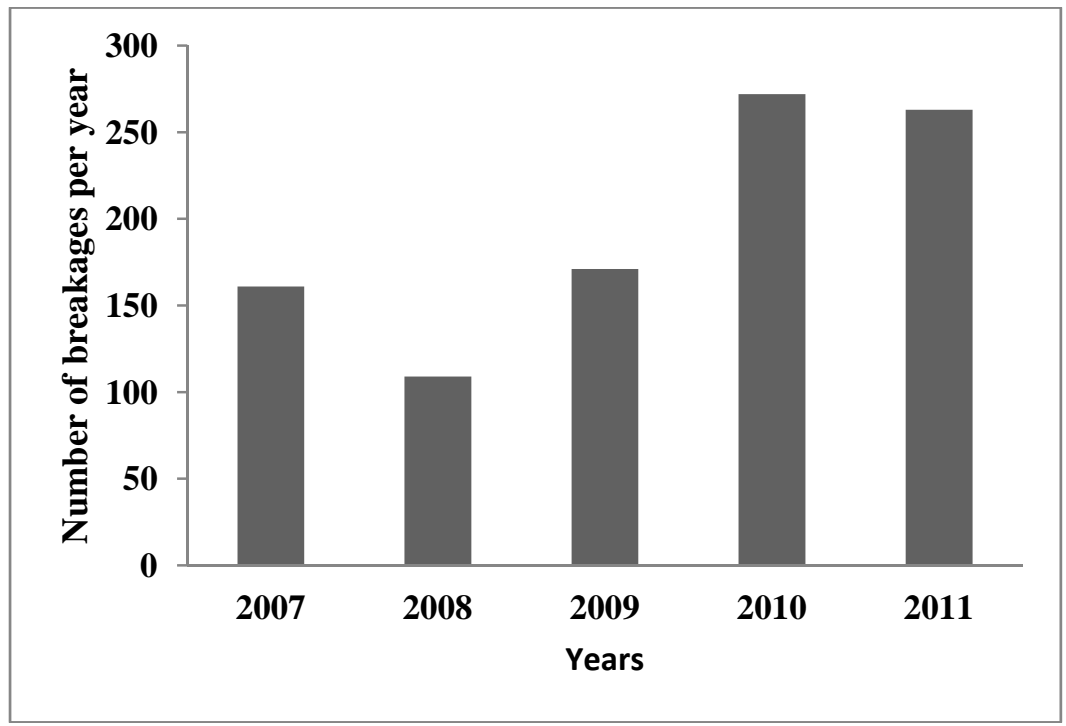

Figure 1: Annual breakages in WPN in the State of Kuwait. 


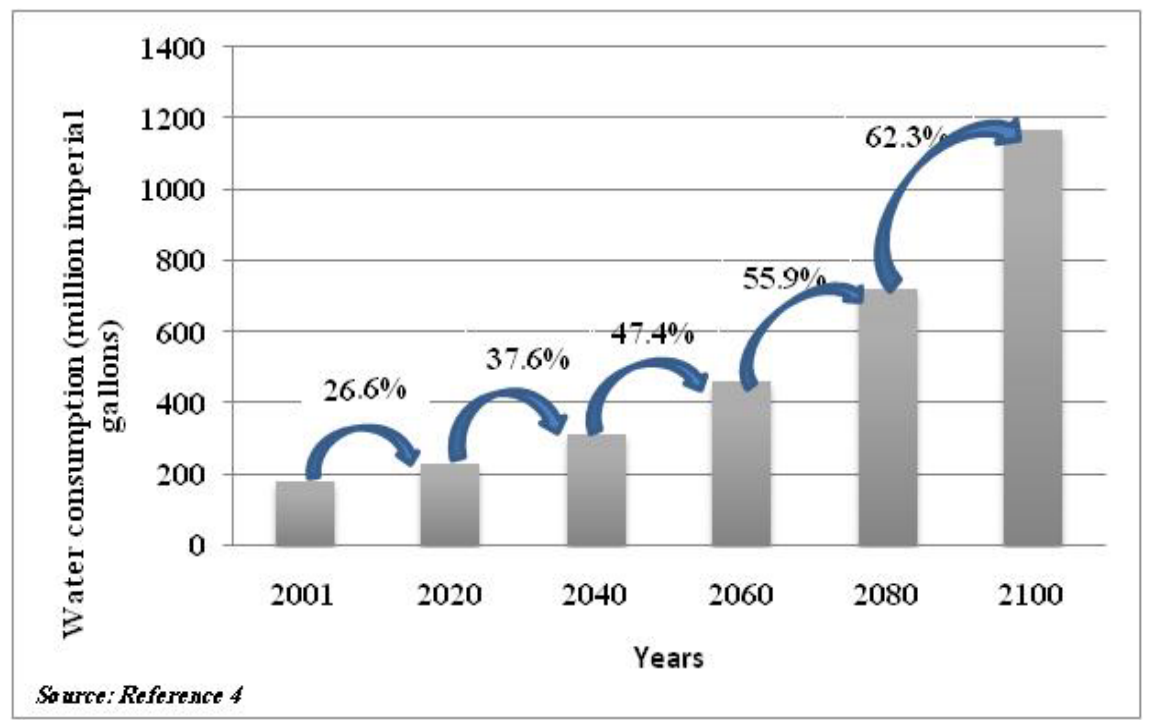

Figure 2: $\quad$ Projected growth of water consumption in the State of Kuwait.

The conclusions drawn from the report on water consumption reveals that the public-supply water continues on an uptrend and the rise is affected by diverse factors, such as the population growth, industrial growth and irrigation. The report further necessitates the discovery of WPN through smart devices to minimize breakages and to monitor the WPN efficiently.

\section{Background of network discovery}

\subsection{Computer network discovery}

Computer network discovery starts by flooding data packets from a known source to remaining entities located at a single hop distance from the source. The hop-by-hop procedure goes repetitive till all the entities at various layers within the network are identified, wherein the identified neighbors at each level are utilized as sources in the consecutive level of discovery. A computer network comprising of various entities such as routers, switches, hubs and end-users is illustrated in Figure 3. A router (assigned with higher ranking in the hierarchy list) with its directly associated entities such as switches, links, hubs and endusers form a domain and the network is divided into various domains. The network discovery identifies and locates the entities by sampling the address and time stamp information corresponding to them. Moreover, the routing tables and address resolution protocols (ARP cache) are also utilized in identifying the active devices. Furthermore, the data retrieved from management information base (MIB) through the simple network management protocol (SNMP) agents is used to provide connectivity information. 


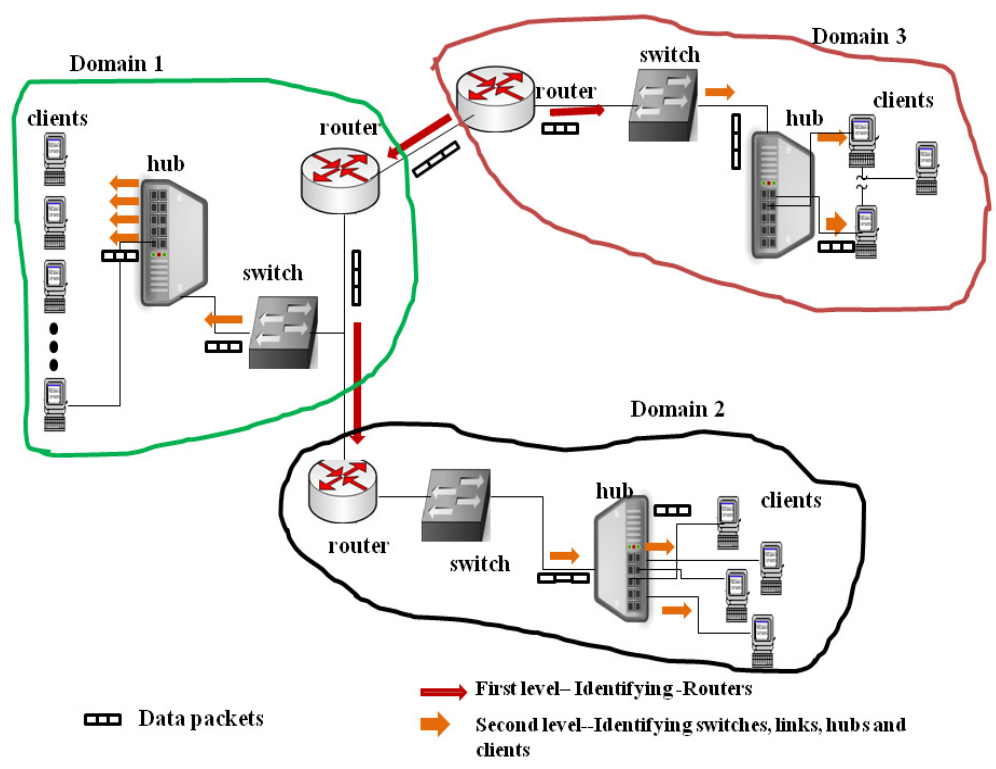

Figure 3: $\quad$ Computer network discovery.

The present network topology discovery tools utilize SNMP in association with MIB to get active device addresses. SNMP is a standard protocol used to collect network management information and MIB is a virtual database used for managing the components of a computer network [1]. We have summarized few researches, which we utilized as a reference to exploit the principle behind the computer network discovery in water pipeline discovery. Burch and Cheswick [5] employed basic internet control message protocol (ICMP) commands like Ping and Traceroute for tracing the topology information. Breitbart et al. [6] proposed an algorithm, which relied only on standard SNMP information to discover layer-2 topology of a heterogeneous, multi subnet network. Researchers in [7, 8], developed novel algorithmic solutions for discovering physical topology in heterogeneous IP networks comprising of multiple IP subnets and various network elements. Their algorithm depended solely on standard information collected in SNMP MIBs. More specifically they utilized information from the complete address forwarding tables (AFT) in Layer-2. Sun et al. [9] proposed a method for deriving a Layer-2 topology and their method was based on knowledge of a root of a spanning tree protocol. Recently Gobijuka and Breitbart [10] have proposed an algorithm for finding layer-2 network topology for a given set of incomplete AFTs in an NP-hard problem. Still research work is going on in this direction to find a better discovery tool to map the entire network at microscopic level.

The time stamp on a packet return from a neighboring node to the source provides an estimate of distance from the source, which is exploited in the discovery of components within WPN. 


\subsection{Proposed water network discovery}

In analogous to computer network, the water pipeline network can also be viewed as a planar network as in Figure 4, whereby the entities such as reservoirs, pipes, valves, elbows and end-user tanks are connected together to form various sectors within WPN. Hereby, the reservoirs are considered as the sources of water flow to flood the WPN.

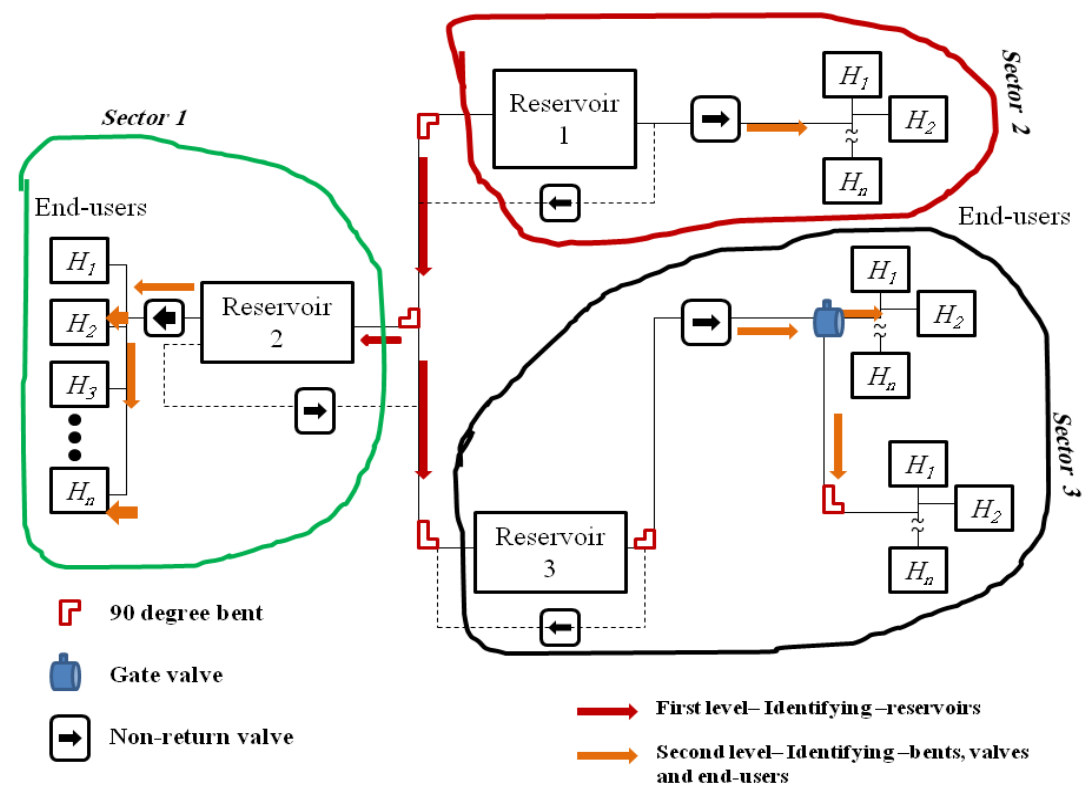

Figure 4: Water pipeline network discovery.

In similarity with the concept of data packet reaching the nearby entity first, the water flow from the reservoir at a constant velocity and constant pressure reaches the entities closer to the source first. The time of emergence of water from an outlet within WPN is found to be analogues with the timestamp on the return data packets in computer network. The timestamp on a data packet depends mainly on bandwidth of the channel and transmission rate, whereas, the time of emergence in WPN is affected by the cross sectional area of the main pipe, the diameter of the outlets and the water flow rate. Furthermore, both in water as well as in computer network, the flow rate should be maintained at certain level to ensure the delivery of water and data packets to the corresponding end-users and outlets respectively.

\subsection{Identifying WPN components}

As a preliminary effort, we have exploited the principle of the computer network discovery in estimating the length of the pipeline network. To start with, we have considered a simple WPN with single inlet and single outlet and with known 
diameter $D$ connected directly to a reservoir, wherein we have tried to deduce the length of the pipeline utilizing the relationship between its volume and pipe diameter. The analysis is further extended to pipeline with single inlet and multiple outlets. The initial assumptions of WPN are: there is no water in the pipe and the cross section of the pipeline is uniform throughout its length. In addition, the water flow rate is constant, and the pressure is also maintained at a constant value. Moreover, the water flow is full of pipes' capacity.

\subsubsection{Pipeline with single inlet and single outlet}

We have utilized the standard equation relating the volume of the pipe with its diameter to estimate pipe length $\mathrm{L}$. The simple horizontal pipeline with single inlet and single outlet satisfying the initial assumptions is shown in Figure 5. The relationship between the volume $\mathrm{V}$ of the flooded water, the cross sectional area $\left(\pi *\left(\frac{D}{2}\right)^{2}\right)$ of the pipeline and its length $\mathrm{L}$ is utilized to estimate the length of the pipeline and it is defined using eqn (1).

$$
L=\left(\frac{2}{D}\right)^{2} * \frac{V}{\pi}
$$

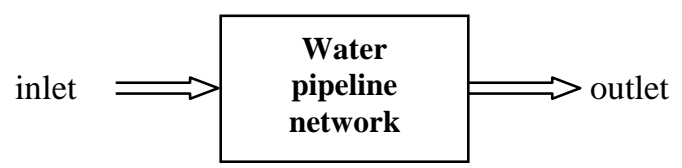

Figure 5: Water pipeline with single outlet and single outlet.

\subsubsection{Pipeline with single inlet and multiple outlets}

Hereby, the length of the pipeline and the position of outlets present in the given pipeline are the parameters to be identified. The pipe with single inlet and multiple outlets is shown in Figure 6, whereby the water flow rate and the water pressure are maintained at a constant value. In analogous to the time stamp in data packets, the time taken by the water to reach out every outlet is utilized to explore the position and the comparative dimensions of the outlets.

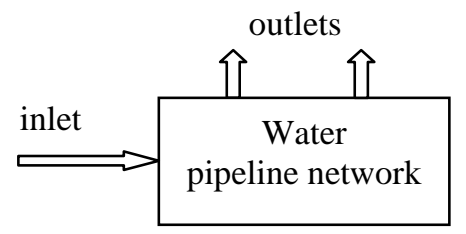

Figure 6: Water pipeline network with two outlets.

Figure 6 is redrawn in Figure 7 to understand the various notations used to represent the dimensions and relative positions of the two outlets with respect to the inlet. The parameter $d_{1}$ denotes the distance from the main inlet to outlet $a$ 
and $d_{2}$ denotes the distance from the main inlet to outlet $b$. Moreover, the two outlets $a$ and $b$ are spaced at a distance of $d_{s}$ and $h_{1}, h_{2}$ are the length of the outlets $a$ and $b$ respectively from the main pipeline. In addition to the initial assumptions, the cross sectional area of the two outlets are considered to be equal.

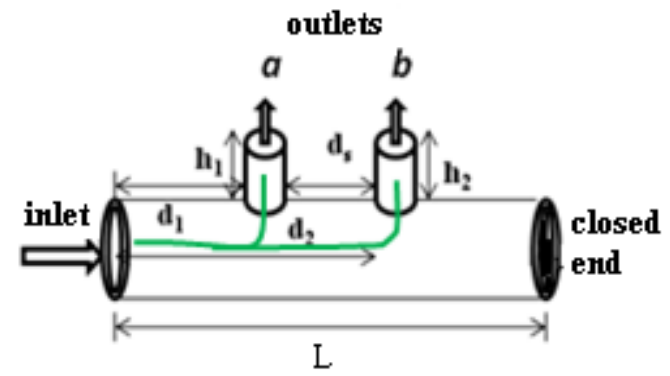

Figure 7: Water pipeline network with two outlets.

We have deduced three categories after observing the time of emergence of the flooded water from the two outlets $a$ and $b$.

Case 1: emergence of water at both the outlets simultaneously

If $\left(d_{1}+h_{1}\right)=\left(d_{2}+h_{2}\right)$, then water will come out from both the outlets simultaneously, wherein, $h_{1}>h_{2}$ and $d_{s}$ is infinitely small. Moreover, if the two outlets are located in opposite direction and are uniformly spaced from the main inlet $\left(d_{1}=d_{2}\right)$, then water will emerge simultaneously in both the outlets.

Case 2: emergence of water at outlet $a$ first and then in outlet $b$

If $d_{1}<d_{2}$ and $h_{1} \leq h_{2}$, then outlet $a$ will disperse water first. This condition implies that the dimensions of length of two given outlets may be equal or the first outlet branch length is of shorter dimensions than the second.

Case 3: emergence of water at outlet $b$ first and then in outlet a

If $\left(d_{1} \sim d_{2}\right) \leq\left(h_{1} \sim h_{2)}\right.$, then outlet $b$ will disperse water first. This shows that the second outlet has shorter length compared to the first outlet.

However, with known spacing between the pipe outlets, we can deduce the total length $x$ of the pipeline using eqn (2), whereby $N$ represents the number of outlets present in the network, $d_{i, i+1}$ is the spacing between $i^{\text {th }}$ and $i+1^{\text {th }}$ outlets, $d_{0}$ is the spacing between the pipe main inlet and the first outlet and $D_{i}$ is the diameter of $i^{\text {th }}$ outlet. If the outlets are equally distributed, then eqn (2) is reduced to eqn (3).

$$
\begin{aligned}
& L=\sum_{i=1}^{N-1} d_{i, i+1}+d_{0}+\sum_{i=1}^{N} D_{i} \\
& L=(N-1) * d+d_{0}+\sum_{i=1}^{N} D_{i}
\end{aligned}
$$




\section{Integrated water network discovery}

This section describes the concept of an integrated WPN discovery system that deploys a bio-inspired legged pipe-crawling robot to discover and validate the layouts of WPN and their interconnections. The current design phase primarily focuses on the development of a novel underwater adhesion system called the Cephalopod-inspired Micro Active Suction Cup Array (MASCA).

\subsection{Background}

The pipe-inspection robots developed recently use a range of different locomotion strategies, among which a combination of wheels and legs is the most common method. In-pipe wheeled robots apply a normal force against the pipe walls to maintain traction and gain propulsion by rotating the wheels [11]. The wheels are typically connected to and controlled by legs in a linkage mechanism with springs that generates a constant normal force, thus resulting in constant friction provided that the interior pipe diameter and surface roughness remains the same. A recent design uses a novel mechanism with permanent magnets to generate a variable normal force to avoid unnecessary high friction and reduce the energy loss [12]. Most in-pipe wheeled robots adapt a design that includes three pairs of wheels 120 degrees apart in the cross-section view to support the robot body at the center of the pipe [13-16]. Most wheeled robots can only fit in a specified pipe size $[13,14]$, while few models can operate within a limited range of varied pipe diameters [15]. The lack of adaptability to different pipe diameters and large cross-sections relative to the pipe interior greatly restrict the operation range of in-pipe wheeled robots. Another imposing challenge for in-pipe robots is power supply. A number of robots are selfcontained with embedded batteries [13], but many other wheeled robots, including commercial models $[16,17]$, are commonly tethered to external power supplies outside the pipe, which allows extended working hours and more embedded electric devices while restricting the operation range by the tether length [14, 15]. Nevertheless, in-pipe wheeled robots generally can maintain sufficient traction and some existing models have the ability to maneuver through up to 90-degree turns, climb up a slope, or even crawl up vertically.

\subsection{Proposed in-pipe robot for WPN discovery}

A bio-inspired legged pipe-crawling robot, as shown in Figure 8, is being developed to address the aforementioned limitations and to ensure functionalities specific to the WPN. A legged robot can potentially have a small cross-section relative to the pipe interior and operate along one side of the pipe interior instead of operating at the pipe center, where the flow rate is the highest. This advantage may allow a legged pipe-crawling robot to be more maneuverable inside a pipe and more accommodating to different sizes of pipes than a wheeled robot. 
The design requirements of this legged in-pipe robot include:

a. Waterproof and not contaminate clean water during operation: All the electric components will be enclosed in the robot body, which has a limited number of kinetic parts to propel the robot. The joints between the body and kinetic parts should prevent water from entering the body. The material that is in contact with water directly should not release any contaminants.

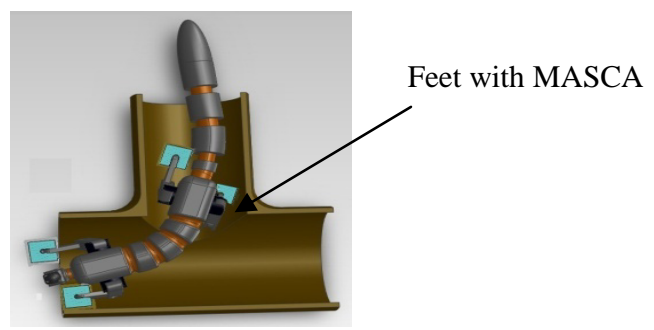

Figure 8: A concept drawing of the bio-inspired legged pipe-crawling robot.

b. High maneuverability in WPN with various pipe diameters and surface roughness: The robot will employ an underwater adhesion system that allows the robot to attach and detach from a surface repeatedly and reliably. The underwater adhesion system will supply sufficient traction for the robot to operate in turbulent water flow of up to $2 \mathrm{~m} / \mathrm{s}$ by using a liquid pump to control an array of micro active suction cups on soft foot pads. The robot will be able to turn up to 90 degrees in the lateral plane.

c. Self-contained with power generation device: The robot will carry a battery and a power generation device. The robot will be designed to be energy efficient, and it can generate power to charge the battery with water flows in the pipe when the battery is low.

The goal of the current design phase is to develop the cephalopod-inspired MASCA on each foot for reliable attachments. The design was inspired by cephalopods, flexible and muscular sessile suckers $[18,19]$. The principle is that a suction cup conforms to a surface and seal against it. The muscle expands the suction cup and causes the enclosed volume to increase, which lowers the internal pressure in the sessile sucker and creates a pressure differential between the interior and exterior of the sucker. This pressure differential applies to the external surface of the suction cup and pushes it against the contact surface.

A proof-of-concept prototype of the robot foot is shown in Figure 9. The prototype consists of three major components, whereby the bottom layer (grey) with the suction cups and diaphragm is made of Dragon Skin ${ }^{\circledR}$ FX-Pro (Smooth-On, Inc.) and the middle layer of the prototype (pink) represents an individual liquid chamber for each suction cup. Further, the top layer (green) creates a liquid chamber on top of all individual chambers and connects them all together. The liquid chamber is then connected to the micro diaphragm liquid pump (KNF Neuberger NF 30 KPDC) via a plastic tube. The middle and top 
layers are made of TAP Silicone RTV (TAP Plastics, Inc.) and also function as structural support for the foot when the pump is activated.

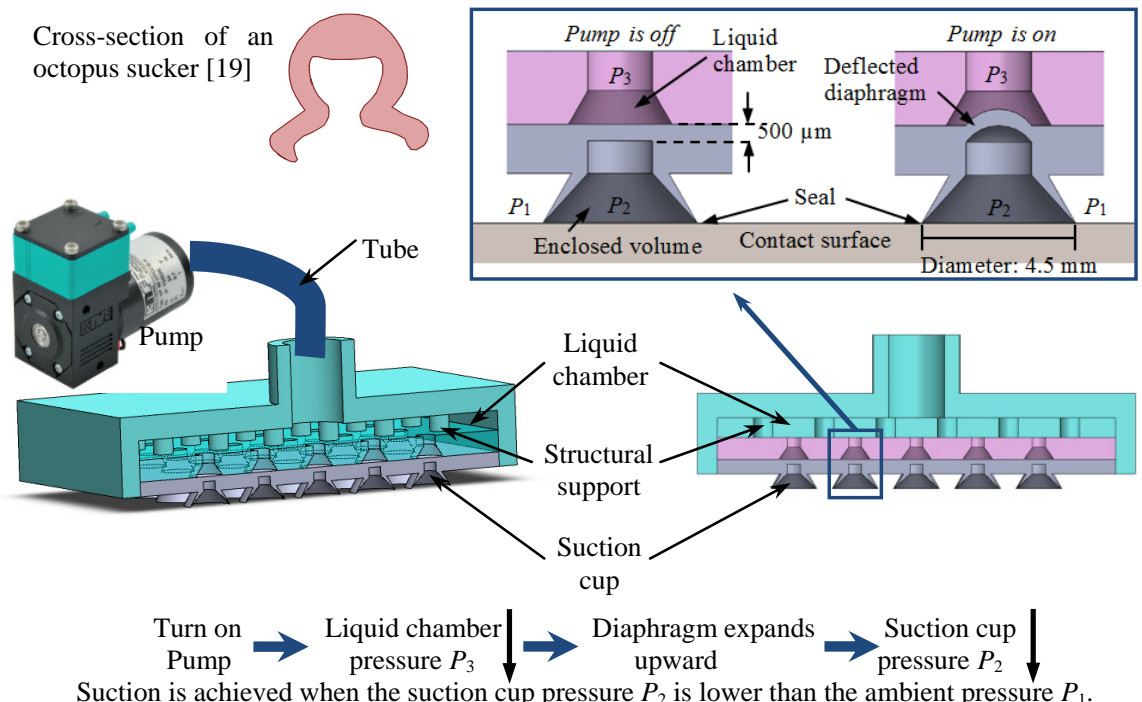

Figure 9: $\quad$ A proof-of-concept prototype.

\section{Conclusion and future work}

In this paper, we present the initial stages of developments of joint research project between Kuwait University and Massachusetts Institute of Technology on developing a bio-inspired crawling robot for water pipeline network (WPN) discovery. We have summarized the current status of WPN in Kuwait and we have exploited the concept behind computer network discovery in deducing the parameters of WPN. Finally, we present the design phase of a novel underwater adhesion system, which grants the maneuverability of the crawling robot in WPN.

\section{Acknowledgement}

The authors would like to acknowledge the support from Kuwait-MIT Center for Natural Resources and the Environment, Kuwait Foundation for the Advancement of Sciences (KFAS) for the research grant no. 2012-5505-01.

\section{References}

[1] http://en.wikipedia.org/.

[2] Statistical Report on Water Network from Statistics Department and Information Center, Kuwait-2007-2011. 
[3] Breitbart, Y., Garofalakis, M., Martin, C., Rastogi, R., Seshadri, S., and Silberschatz, A., Topology Discovery in Heterogeneous IP Networks. Proc. of Nineteenth Annual Joint Conference of the IEEE Computer and Communications Societies. 26-30 March 2000, Tel Aviv, Israel, vol.1, pp.265-274, 2000.

[4] Ghunaimi, Z., Water Security in Kuwait and Arabian Gulf Countries, published by Center for Research and Studies on Kuwait, Kuwait, 2005.

[5] Burch, H., and Cheswick, B., Mapping the internet, IEEE Computer, vol. 32, issue 4, pp 97-98,1999.

[6] Breitbart, Y., Minos, G., Ben, J., Cliff, M., Rajeev, R., and Silberschatz, A., Topology Discovery in Heterogeneous IP networks: The NetInventory System, IEEE/ACM Transactions on Networking, vol.12, issue, 3, pp.401414, 2004.

[7] Gobijuka, H., and Breitbart, Y., Discovering Network Topology of Large Multisubnet Ethernet Networks. Proc. of IEEE Local Computer Networks, 15-18 October 2007, Dublin, Ireland, pp 428-435.

[8] Lowekamp, B., O’Hallaron, D. R., Gross, T. R., Topology Discovery for Large Ethernet Networks. Proc. of ACM SIGCOMM, 27-31 August 2001, San Diego, CA, USA, pp. 237-248.

[9] Sun, Y., Shi, Z., and Wu, Z., A Discovery Algorithm for Physical Topology in Switched Networks, Proc. of IEEE Conference on Local Computer Networks, 17 November 2005, Sydney, Australia, pp.311-317.

[10] Gobijuka, H., and Breitbart, Y., Ethernet Topology Discovery for Networks with Incomplete Information. IEEE/ACM Transactions on Networking, vol.18, issue 4, pp.1220-1233, 2010.

[11] Suzumori, K., Miyagawa, T., Kimura, M., and Hasegawa, Y., Micro Inspection Robot for 1-in Pipes, Mechatronics, IEEE/ASME Transactions on , vol.4, no.3, pp.286-292, Sep 1999.

[12] Changrak, C., Chatzigeorgiou, D., Ben-Mansour, R., and Youcef-Toumi, K., Design and Analysis of Novel Friction Controlling Mechanism with Minimal Energy for In-pipe Robot Applications, Proc. of IEEE International Conference on Robotics and Automation , 14-18 May 2012, St. Paul, MN, USA, pp.4118-4123.

[13] Jung-Sub, L., Se-Gon, R., Do Wan, K., Hyungpil, M., and Hyouk, R.C., Inpipe Robot Navigation Based on the Landmark Recognition System using Shadow Images. Proc. of the IEEE International Conference on Robotics and Automation. 12-17 May 2009, Kobe, Japan, pp. 3441-3446.

[14] Peng, L., Shugen, Ma., Bin, L., Yuechao, Wang., and Yunhui, Liu., Selfrescue Mechanism for Screw Drive In-pipe Robots, Proc. of IEEE/RSJ International Conference on Intelligent Robots and Systems, 18-22 October 2010, pp.2843-2849.

[15] Zhang, Y., and Yan, G., In-pipe Inspection Robot with Active Pipediameter Adaptability and Automatic Tractive Force Adjusting, Mechanism and Machine Theory, vol. 42, issue 12, pp. 1618-1631, 2007.

[16] Internal Robotic inspection Tool for Unpiggable Pipe Inspections, 
(http://www.geindustrial.com/publibrary/checkout/Robotics?TNR=Articles| Robotics|generic)

[17] PureRobotics - Robotic Pipeline Inspection (http://www.puretechltd.com/ services/robotics/)

[18] Smith, A. M., Cephalopod Sucker Design and the Physical Limits to Negative Pressure, Journal of Experimental Biology, vol. 199, pp. 949-958, 1996.

[19] Kier, W. M., and Smith, A. M., The Structure and Adhesive Mechanism of Octopus Suckers. Integrative and Comparative Biology, 2-6 January, Anaheim, CA, USA, pp.1146-1153, 2002. 